\title{
SUCESSÃO EM FRAGMENTOS FLORESTAIS ALTOMONTANOS NO SUL DO BRASIL: UMA ABORDAGEM FLORÍSTICO-ESTRUTURAL E FILOGENÉTICA
}

\section{SUCCESSION IN HIGHLAND FOREST FRAGMENTS IN SOUTH BRAZIL: A FLORISTIC- STRUCTURAL AND PHYLOGENETIC APPROACH}

\begin{abstract}
Edilaine Duarte ${ }^{1}$ Ana Carolina da Silva ${ }^{2}$ Pedro Higuchi ${ }^{2}$ Janaina Gabriela Larsen $^{3}$ Danielle Cristina Ortiz ${ }^{1}$ Aline Gross ${ }^{1}$, Eliana Turmina ${ }^{1}$ Jéssica Thalheimer Aguiar $^{1}$ Manoela Bez Vefago ${ }^{1}$ Chaiane Rodrigues Schneider ${ }^{1}$ Silvane de Fátima Siqueira ${ }^{4}$ Angela Camila Lemos ${ }^{4}$ Lirio Ribeiro $^{5}$
\end{abstract}

\begin{abstract}
RESUMO
Objetivou-se caracterizar a organização florístico-estrutural, a riqueza e a estrutura filogenética de comunidades arbóreas de fragmentos florestais altomontanos em diferentes estágios sucessionais, situados no Parque Nacional de São Joaquim em Urubici, Santa Catarina. Foram selecionados três fragmentos, dois em avançado estágio sucessional e um em estágio inicial, sendo cada um avaliado por meio de 20 parcelas de $10 \times 10$ m, distribuídas na forma de transecção de 20 x 100 m. Foram identificados e medidos todos os indivíduos arbóreos com DAP $\geq 5 \mathrm{~cm}$. Os dados foram analisados por meio de rarefação, ordenação multivariada, análise de espécies indicadoras e métricas de estruturação filogenética. A riqueza encontrada em cada fragmento não apresentou relação com o estágio sucessional, uma vez que as áreas não difeririam quanto a este aspecto. A organização florístico-estrutural foi influenciada pela interação entre o estágio sucessional e as diferenças ambientais entre os locais. Na área em início de sucessão, foi observado o agrupamento filogenético das espécies, e nas áreas tardias, o padrão foi aleatório. Assim, os resultados evidenciaram variações na riqueza independentemente do estágio sucessional e que a sucessão florestal influenciou a composição florístico-estrutural e a estrutura filogenética das áreas.
\end{abstract}

Palavras-chave: ecologia florestal; filogenia; Floresta Ombrófila Mista Alto-Montana.

\section{ABSTRACT}

We aimed to describe the floristic-structural organization, the richness and the phylogenetic structure of tree components in highland forest fragments in different successional stages, at São Joaquim National Park, in the municipality of Urubici, Santa Catarina state. Three fragments were selected, two in an advanced successional stage and one in an initial successional stage, being each one evaluated by $2010 \mathrm{x} 10 \mathrm{~m}$ sampling plots, allocated as $20 \times 100 \mathrm{~m}$ transects. All trees with $\mathrm{dbh} \geq 5 \mathrm{~cm}$ were identified and measured. The data were analyzed through rarefaction, multivariate ordination, indicator species analysis and phylogenetic structure metrics. There was no relationship between the richness and the successional stage,

1 Engenheira Florestal, MSc., Departamento de Engenharia Florestal, Universidade do Estado de Santa Catarina, Av. Luiz de Camões, 2090, CEP 88520-000, Lages (SC), Brasil. ediduarte10@gmail.com / ortiz.dco@gmail.com / alinegros@hotmail.com / eliana_turmina@hotmail.com / jeh.aguiar93@hotmail.com / manuvefago@gmail.com / chai.rodriguesschneider@gmail.com

2 Engenheiro Florestal, Dr., Professor do Departamento de Engenharia Florestal, Universidade do Estado de Santa Catarina, Av. Luiz de Camões, 2090, CEP 88520-000, Lages (SC), Brasil. carol_sil4@yahoo.com.br / higuchip@ gmail.com

3 Graduanda em Engenheiro Florestal, Departamento de Engenharia Florestal, Universidade do Estado de Santa Catarina, Av. Luiz de Camões, 2090, CEP 88520-000, Lages (SC), Brasil. janainalarsen18@gmail.com

4 . Bióloga, MSc., Departamento de Engenharia Florestal, Universidade do Estado de Santa Catarina, Av. Luiz de Camões, 2090, CEP 88520-000, Lages (SC), Brasil. biosilvane@hotmail.com / angelacl@ymail.com

5 Engenheiro Florestal, Departamento de Engenharia Florestal, Universidade do Estado de Santa Catarina, Av. Luiz de Camões, 2090, CEP 88520-000, Lages (SC), Brasil. lirioribeiro@yahoo.com.br

Recebido para publicação em 8/03/2017 e aceito em 8/08/2017

Ci. Fl., v. 28, n. 3, jul. - set., 2018 
once the studied areas did not differ in relation to the number of species. The floristic-structural organization was influenced by the interaction between the successional stage and the environmental differences among areas. Phylogenetic clustering of species was observed in the initial successional stage area and random phylogenetic pattern was observed in advanced successional stage areas. The results indicated independent variation in richness in relation to successional phase and, that the forest succession influenced the floristicstructural composition and phylogenetic structure of the study areas.

Keywords: forest ecology; phylogeny; upper-montane Araucaria Forest.

\section{INTRODUÇÃO}

Devido ao histórico de fragmentação e degradação dos ecossistemas florestais tropicais e subtropicais, estudos sobre o processo de sucessão florestal secundária nestas áreas se tornam fundamentais, pois embasam inferências sobre a resiliência de ambientes perturbados e podem subsidiar estratégias de restauração florestal. Nesse sentido, as pesquisas sobre os processos de organização de comunidades de espécies vegetais ao longo do tempo contribuem para a compreensão de como ocorre a trajetória sucessional pós-distúrbio. Estas têm sido o foco de vários estudos em diferentes partes do mundo (GUARIGUATA; OSTERTAG, 2001; CHAZDON, 2008), inclusive no Brasil (ZANINI et al., 2014; SCHWEIZER et al., 2015; MARCILIO-SILVA; PILLAR; MARQUES, 2016), onde restam cerca de 7\% da área original de Floresta Atlântica (VARJABEDIAN, 2010).

O estágio sucessional pode ser definido por aspectos relacionados à composição de espécies, biomassa total e tamanho de populações, com variações em escala espacial e temporal (BEGON; TOWNSEND; HARPER, 2006). A sucessão florestal secundária é aquela que ocorre após a remoção parcial da vegetação natural, com a manutenção de material orgânico e/ou propágulos da vegetação original na área, e é sintetizada por Chazdon (2008) como sendo um processo complexo, controlado pela interação de fatores determinísticos e estocásticos, com uma rápida recuperação estrutural e de riqueza, e um lento restabelecimento da composição florística. Ainda, conforme a mesma autora, em áreas distintas, a recuperação da flora durante a sucessão não ocorre de forma convergente, uma vez que é condicionada por condições locais, como o histórico de perturbação, as condições edáficas e a distância da fonte de propágulos, o que torna a predição das mudanças florísticas ao longo da trajetória sucessional uma tarefa complexa (GUARIGUATA; OSTERTAG, 2001). Esse avanço sucessional é dependente do processo de colonização de árvores pioneiras e das características ambientais, que irão propiciar o estabelecimento de espécies tardias, restabelecendo os processos ecológicos (WIRTH et al., 2009).

Recentemente, estudos com uma abordagem filogenética têm sido realizados com o propósito de explicar a ocorrência de espécies em comunidades biológicas (e.g. GERHOLD et al., 2015; BELLO et al., 2017; DEXTER et al., 2017), inclusive no contexto do processo de sucessão florestal (e.g. LETCHER et al., 2012; MARCILIO-SILVA; PILLAR; MARQUES, 2016). Estes estudos são baseados na premissa de que a distância filogenética entre as espécies pode refletir as diferenças ecológicas das mesmas, considerando que atributos funcionais podem ser filogeneticamente conservados (WEBB, 2000; WEBB et al., 2002; CIANCIARUSO; SILVA; BATALHA, 2009; BELLO et al., 2017). No contexto de sucessão, alguns estudos (e.g. LETCHER et al., 2012; MARCILIO-SILVA; PILLAR; MARQUES, 2016) têm demonstrado maior agrupamento filogenético das espécies em fases iniciais e maior distanciamento nas fases tardias. Porém, outros estudos observaram padrões distintos de estruturação filogenética ao longo do tempo (e.g. CHANG; ZHOU; PENG, 2015; SCHWEIZER et al., 2015), o que demonstra a complexidade do processo de sucessão, que não pode ser generalizado. Neste contexto, a relação entre as guildas de regeneração, que refletem atributos funcionais específicos das espécies, e a estrutura filogenética de comunidades, pode fornecer importantes informações sobre os fatores ecológicos determinantes no processo sucessional.

Desta forma, o presente estudo teve como principal objetivo caracterizar a riqueza, a organização florístico-estrutural e a estruturação filogenética do componente arbóreo em áreas florestais que se encontram em diferentes fases sucessionais, em florestas altomontanas no sul do Brasil. Esta região se destaca por apresentar uma composição florística peculiar, resultante da existência de fatores ambientais limitantes, tais como temperaturas baixas, elevada umidade relativa do ar, baixa incidência de radiação solar e solos geralmente pouco profundos, que refletem um ambiente restritivo de espécies (HIGUCHI et al., 2013). Do 
ponto visto ambiental, estas florestas, que se encontram ameaçadas em função de um cenário de mudanças climáticas e estão sujeitas a perturbações antrópicas crônicas, desempenham importante funções, como a proteção do solo, manutenção do ciclo hidrológico e sequestro de carbono (HIGUCHI et al., 2012). Testouse a hipótese de que o fragmento em fase inicial apresenta menor número de espécies, uma organização florístico-estrutural distinta e um agrupamento filogenético das espécies coocorrentes em função da maior participação de espécies pioneiras. Espera-se, com o presente trabalho, contribuir para o entendimento do processo de estabelecimento da vegetação em ambientes altomontanos e subsidiar estratégias de restauração florestal neste importante ecossistema.

\section{MATERIAL E MÉTODOS}

O estudo foi realizado em três diferentes áreas florestais no Parque Nacional de São Joaquim, Planalto Catarinense, que se encontravam em fases sucessionais distintas. A área do parque, criado em 1961, abrange alguns municípios do estado de Santa Catarina, sendo os locais de estudo localizados no município de Urubici-SC. O clima da região é do tipo $\mathrm{Cfb}$ de acordo com a classificação de Köppen. A precipitação média anual é de $1.753 \mathrm{~mm}$ e a temperatura média anual é de $12,7^{\circ} \mathrm{C}$, segundo dados do WorldClim (HIJMANS et al., 2005).

A vegetação é um mosaico de campos e Floresta Ombrófila Mista Alto-Montana (IBGE, 2012), formando fragmentos florestais em uma matriz campestre. Além disso, devido a processos de desmatamentos no passado, antes da desapropriação das áreas do Parque, houve também a fragmentação antrópica. Os solos são classificados como Neossolos Litólicos e Organossolos, e o relevo varia de suave ondulado a fortemente ondulado.

A partir de observações no campo e entrevistas com funcionários do parque, as áreas de estudo foram classificadas em dois estágios distintos de sucessão: inicial e avançado. As áreas em avançado estágio de sucessão [Estágio Avançado $1(8,50 \mathrm{ha})=28^{\circ} 05^{\prime} 41,5^{\prime}$ 'S e 49³0'14,71”W; Estágio Avançado 2 (1,5 ha) $=28^{\circ} 09^{\prime} 49,19^{\prime \prime} \mathrm{S}$ e $\left.49^{\circ} 36^{\prime} 47,56^{\prime \prime} \mathrm{W}\right]$, com altitude aproximada de $1.600 \mathrm{~m}$, apresentam, principalmente, espécies secundárias e tardias, como, por exemplo, Drimys angustifolia Miers e Siphoneugena reitzii D.Legrand (Figuras 1a e 1b). Por meio da observação no campo, pode-se perceber que ambas as áreas em avançado estágio de sucessão apresentam uma fisionomia do componente arbóreo caracterizada por um dossel predominantemente fechado, com árvores alcançando aproximadamente $10 \mathrm{~m}$, com ausência de indícios de degradação, de forma que as mesmas podem ser consideradas como bem estruturadas e conservadas. Por meio das mesmas observações de campo, também foi observado que na área "Estágio Avançado 1" existia uma maior heterogeneidade da vegetação, devido à presença de algumas clareiras. Destaca-se, também, a presença constante de nuvens, caracterizando-as como florestas nebulares. Na área "Estágio Avançado 2" existe, ainda, a presença de um pequeno curso d'água (cerca de $50 \mathrm{~cm}$ de largura). Assim, as áreas avaliadas também apresentam elevada heterogeneidade ambiental, que foram consideradas na interpretação dos resultados. A área em fase inicial de sucessão [Estágio Inicial $(9,5 \mathrm{ha})=28^{\circ} 04^{\prime} 46,87^{\prime}$ 'S e 49³0'51,29'W], com altitude aproximada de $1.350 \mathrm{~m}$, devido ao histórico de uso do solo e manejo, é composta basicamente de espécies pioneiras (Figura 1c), adaptadas a pleno sol. Conforme entrevistas realizadas com os funcionários do parque, esta área foi sujeita a desmatamentos e corte seletivos até a década de 1980, com o propósito de uso para pastagem, sendo integralmente protegida em 2008. 

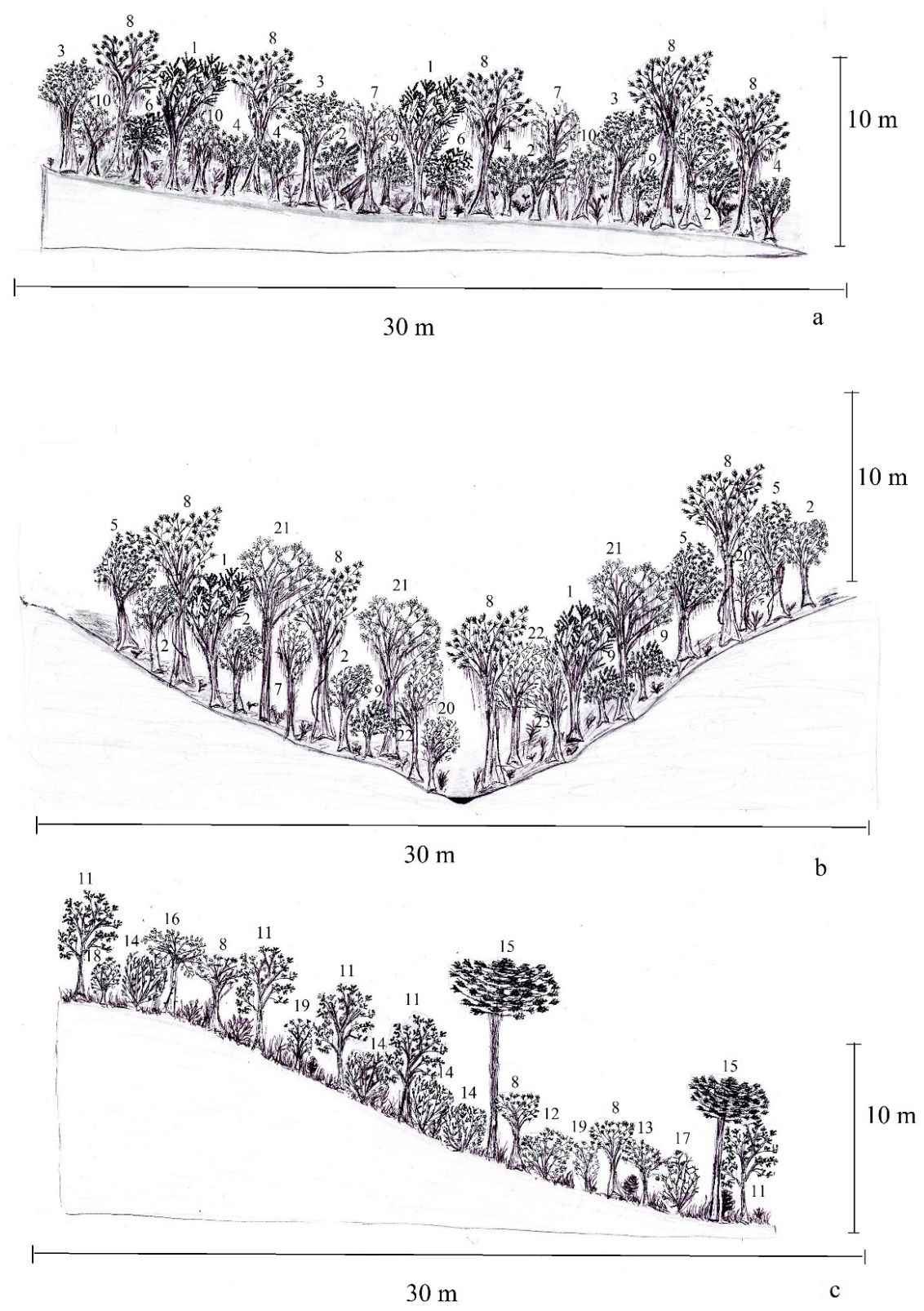

Weinmannia paulliniifolia Pohl ex Ser. (1), Myrcengenia oxysepala (Burret) D.Legrand \& Kausel (2), Eugenia uruguayensis Cambess. (3), Myrceugenia mesomischa (Burret) D.Legrand \& Kausel(4), Siphoneugena reitzii D.Legrand (5), Dicksonia sellowiana Hook. (6), Prunus myrtifolia (L.) Urb. (7), Drimys angustifolia Miers (8), Myrceugenia regnelliana (O.Berg) D.Legrand \& Kausel (9), Myrceugenia myrcioides (Cambess.) O.Berg (10), Mimosa scabrella Benth. (11), Myrceugenia euosma (O.Berg) D.Legrand (12), Baccharis microdonta DC. (13), Baccharis uncinella DC. (14), Araucaria angustifolia (Bertol.) Kuntze (15), Vernonanthura discolor (Spreng.) H.Rob. (16), Campovassouria cruciata (Vell.) R.M.King \& H.Rob. (17), Solanum compressum L.B.Sm. \& Downs (18), Symphyopappus itatiayensis (Hieron.) R.M.King \& H.Rob. (19), Myrceugenia glaucescens (Cambess.) D.Legrand \& Kausel (20), Crinodendron brasiliense Reitz \& L.B.Sm. (21), Ilex microdonta Reissek (22) e Ocotea pulchella (Nees \& Mart.) Mez (23).

FIGURA 1: Perfil esquemático de três fragmentos florestais, em diferentes estágios sucessionais $(\mathrm{a}=$ Estágio Avançado 1;b=Estágio Avançado 2; $\mathrm{c}=$ Estágio Inicial), em Urubici-SC, com as espécies representativas.

FIGURE 1: Schematic profile of three forest fragments, in different successional stages ( $\mathrm{a}=$ Advanced Stage 1; $\mathrm{b}$ $=$ Advance Stage 2; $\mathrm{c}=$ Initial Stage), in the municipality of Urubici, SC state, with its representative species. 
Para o levantamento florístico-estrutural, em cada área foram alocadas 20 parcelas, de 10 x $10 \mathrm{~m}$, distribuídas em transecções de $20 \times 100 \mathrm{~m}$. Cada transecção foi alocada na direção do fragmento florestal e adjacente à matriz campestre, de forma perpendicular à borda. $\mathrm{Na}$ área em estágio inicial, por apresentar formato irregular, foi necessária a divisão da transecção em duas de $20 \times 50 \mathrm{~m}$, ambas adjacentes à área de vegetação aberta. Para verificar se a amostragem foi adequada para caracterizar a composição florística das áreas de estudo, foram geradas curvas de acumulação de espécies, por meio do método de rarefação, uma para cada área.

Em cada parcela foram coletados, identificados, marcados com plaquetas de alumínio e medidos (CAP - circunferência a altura do peito - e estimada a altura) todos os indivíduos arbóreos com DAP (diâmetro altura do peito, medido a 1,30) $\geq 5 \mathrm{~cm}$. Indivíduos que apresentarem troncos múltiplos foram medidos quando a raiz da soma dos quadrados dos DAPs foi $\geq 5 \mathrm{~cm}$. As identificações das espécies foram realizadas por meio de comparações em literatura especializada e consulta a especialistas. As espécies foram classificadas nas famílias de acordo com o sistema APG III (ANGIOSPERM PHYLOGENY GROUP, 2009) e incorporadas ao Herbário LUSC da Universidade do Estado de Santa Catarina.

A riqueza entre as áreas foi comparada utilizando-se curvas de rarefação para padronização do número de indivíduos e um diagrama de Venn. Com o propósito de encontrar agrupamentos florísticoestruturais entre as áreas de estudo, associados a diferentes estágios de sucessão florestal, foi realizada uma análise multivariada NMDS (Non-metric Multidimensional Scaling). Para detectar as espécies mais importantes em cada fragmento, foi realizada a Análise de Espécies Indicadoras (DUFRANE; LEGENDRE, 1997).

A partir do banco de dados de levantamentos vegetacionais do Laboratório de Dendrologia e Fitossociologia da UDESC, construído a partir de publicações em revistas científicas (https://doi.org/10.6084/ m9.figshare.5139907.v1), foi elaborada uma lista florística de árvores ocorrentes em áreas altomontanas, representando, assim, o conjunto regional (pool) de espécies. Gimnospermas e pteridófitas arborescentes foram excluídas por serem muito distantes filogeneticamente das angiospermas, uma vez que representariam um viés na análise. Por meio desta lista, foi construída uma árvore filogenética, utilizando o PHYLOMATIC 3 (WEBB; DONOGHUE, 2004), junto com a mega-árvore R20120829mod.new, disponibilizada por Gastauer e Meira Neto (2016). Os comprimentos dos ramos da árvore filogenética foram definidos a partir do algoritmo BLADJ, disponibilizado pelo programa PHYLOCOM (WEBB; ACKERLY; KEMBEL, 2008) e da estimativa de idades dos clados (ages_logn), disponibilizada por Gastauer e Meira Neto (2016), com base nos trabalhos de Hedges e Kumar (2009) e Bell, Soltis e Soltis (2010). Para determinar a estrutura filogenética, foram calculadas as medidas de diversidade baseadas na árvore filogenética construída, para cada componente de cada área, considerando os dados de abundância: i) MPD (Mean phylogenetic distance), que quantifica as relações filogenéticas dos indivíduos em cada componente de cada área, por meio da distância média entre os pares de indivíduos; e ii) NRI (Net relatedness index), que compara o valor de MPD observado nos componentes das áreas com o valor de MPD esperado para o conjunto regional de espécies (WEBB; ACKERLY; KEMBEL, 2008). Para isto, utilizou-se o algoritmo independentswap (GOTELLI, 2000) como modelo nulo, para geração de 1.000 comunidades aleatórias. Valor positivo de NRI e valores de MPDs observados inferiores aos MPDs simulados por 975 vezes indica que as espécies são mais próximas filogeneticamente do que o esperado ao acaso (agrupamento filogenético). Quando os valores de NRI são negativos e os valores de MPDs observados são superiores aos MPDs simulados por 975 vezes, as espécies são filogeneticamente mais distantes, resultando em dispersão filogenética (WEBB, 2000). Considerando-se que a resolução de árvores filogenéticas pode influenciar o cálculo de métricas de estruturação filogenética de comunidades biológicas, a mesma foi avaliada conforme o trabalho de Swenson (2009).

Além do uso do PHYLOMATIC (WEBB; DONOGHUE, 2004) e PHYLOCOM (WEBB; ACKERLY; KEMBEL, 2008), as análises foram realizadas utilizando-se planilha eletrônica e a linguagem de programação estatística R (R DEVELOPMENT CORE TEAM, 2016), com os pacotes vegan (OKSANEN et al., 2009) para a rarefação e ordenação NMDS, labdsv (ROBERTS, 2016) para a análise de espécies indicadoras e picante (KEMBEL et al., 2010) para a análise de estruturação filogenética. 


\section{RESULTADOS E DISCUSSÃO}

Nas três áreas avaliadas, foram amostrados 895 indivíduos, distribuídos em 18 famílias, 27 gêneros e 43 espécies (Tabela 1). Quando comparados com estudos na região realizados em pisos altitudinais inferiores (e.g. NASCIMENTO; LONGHI; BRENA, 2001; HIGUCHI et al., 2012), observa-se que as áreas avaliadas apresentaram baixa riqueza de espécies. Além disso, destaca-se o elevado número de indivíduos de Crinodendron brasiliense Reitz \& L.B.Sm. (47) encontrado na área "Estágio Avançado 2", espécie endêmica das regiões de elevada altitude do Planalto Sul Catarinense.

TABELA 1: Espécies amostradas em três fragmentos florestais em Urubici-SC, em diferentes estágios sucessionais (A1 = Estágio Avançado 1; A2 = Estágio Avançado 2; I = Estágio Inicial).

TABLE 1: Sampled species in three forest fragments in the municipality of Urubici, SC state, in different successional stages $(\mathrm{A} 1=$ Advanced Stage $1 ; \mathrm{A} 2=$ Advanced Stage 2; I = Initial Stage $)$.

\begin{tabular}{|c|c|c|c|c|}
\hline Famílias/Espécies & A1 & $\mathrm{A} 2$ & $\mathrm{I}$ & Voucher \\
\hline \multicolumn{5}{|l|}{ Anacardiaceae } \\
\hline Schinus polygamus (Cav.) Cabrera & & 1 & 1 & 9051 \\
\hline \multicolumn{5}{|l|}{ Aquifoliaceae } \\
\hline Ilex microdonta Reissek & 11 & 9 & & 9034 \\
\hline Ilex paraguariensis A.St.-Hil. & & & 1 & 9035 \\
\hline \multicolumn{5}{|l|}{ Araucariaceae } \\
\hline Araucaria angustifolia (Bertol.) Kuntze & & 1 & 9 & - \\
\hline \multicolumn{5}{|l|}{ Asteraceae } \\
\hline Baccharis dentata (Vell.) G.M.Barroso & 4 & 2 & & 9023 \\
\hline Baccharis erioclada DC. & & & 1 & 9024 \\
\hline Baccharis lateralis Baker & 1 & & & 9025 \\
\hline Baccharis microdonta DC. & & & 1 & 8731 \\
\hline Baccharis oblongifolia (Ruiz \& Pav.) Pers. & 1 & & & 9026 \\
\hline Baccharis uncinella DC. & & & 26 & 9027 \\
\hline $\begin{array}{l}\text { Campovassouria cruciata (Vell.) } \\
\text { R.M.King \& H.Rob. }\end{array}$ & & & 5 & \\
\hline $\begin{array}{l}\text { Symphyopappus itatiayensis (Hieron.) } \\
\text { R.M.King \& H.Rob. }\end{array}$ & 7 & 2 & 1 & 9057 \\
\hline Vernonanthura discolor (Spreng.) H.Rob. & 1 & & 4 & 9058 \\
\hline \multicolumn{5}{|l|}{ Berberidaceae } \\
\hline Berberis laurina Billb. & 2 & & & 9028 \\
\hline \multicolumn{5}{|l|}{ Cardiopteridaceae } \\
\hline Citronella paniculata (Mart.) R.A.Howard & 7 & & & 9030 \\
\hline \multicolumn{5}{|l|}{ Celastraceae } \\
\hline Maytenus boaria Molina & 2 & 1 & & 9036 \\
\hline \multicolumn{5}{|l|}{ Cunoniaceae } \\
\hline Weinmannia paulliniifolia Pohl ex Ser. & 22 & 13 & & 9059 \\
\hline \multicolumn{5}{|l|}{ Dicksoniaceae } \\
\hline Dicksonia sellowiana Hook. & 14 & & & - \\
\hline \multicolumn{5}{|l|}{ Elaeocarpaceae } \\
\hline Crinodendron brasiliense Reitz \& L.B.Sm. & 1 & 47 & & 9031 \\
\hline Fabaceae & & & & \\
\hline
\end{tabular}


TABELA 1: Continuação...

TABLE 1: Continued...

\begin{tabular}{|c|c|c|c|c|}
\hline Famílias/Espécies & A1 & $\mathrm{A} 2$ & $\mathrm{I}$ & Voucher $^{1}$ \\
\hline Mimosa scabrella Benth. & & & 116 & 9037 \\
\hline \multicolumn{5}{|l|}{ Lauraceae } \\
\hline $\begin{array}{l}\text { Cinnamomum amoenum (Nees \& Mart.) } \\
\text { Kosterm. }\end{array}$ & & & 2 & - \\
\hline Ocotea porosa (Nees \& Mart.) Barroso & 3 & & & 9047 \\
\hline Ocotea pulchella (Nees \& Mart.) Mez & 5 & 10 & & 9048 \\
\hline Persea willdenovii Kosterm. & 2 & & & 9049 \\
\hline \multicolumn{5}{|l|}{ Myrtaceae } \\
\hline Blepharocalyx salicifolius (Kunth) O.Berg & 1 & & & - \\
\hline Eugenia uruguayensis Cambess. & 15 & & & 9033 \\
\hline Myrceugenia euosma (O.Berg) D.Legrand & 1 & & 3 & 9038 \\
\hline $\begin{array}{l}\text { Myrceugenia glaucescens (Cambess.) } \\
\text { D.Legrand \& Kausel }\end{array}$ & 2 & 9 & & 9039 \\
\hline $\begin{array}{l}\text { Myrceugenia mesomischa (Burret) } \\
\text { D.Legrand \& Kausel }\end{array}$ & 20 & & & 9040 \\
\hline $\begin{array}{l}\text { Myrceugenia miersiana (Gardner) } \\
\text { D.Legrand \& Kausel }\end{array}$ & 10 & & & 9041 \\
\hline $\begin{array}{c}\text { Myrceugenia myrcioides (Cambess.) } \\
\text { O.Berg }\end{array}$ & 42 & & & 9042 \\
\hline $\begin{array}{l}\text { Myrceugenia oxysepala (Burret) } \\
\text { D.Legrand \& Kausel }\end{array}$ & 28 & 11 & & 9043 \\
\hline $\begin{array}{l}\text { Myrceugenia regnelliana (O.Berg) } \\
\text { D.Legrand \& Kausel }\end{array}$ & 16 & 33 & & 9044 \\
\hline Myrrhinium atropurpureum Schott & 3 & & & 9045 \\
\hline Siphoneugena reitzii D.Legrand & 13 & 64 & & 9052 \\
\hline \multicolumn{5}{|l|}{ Primulaceae } \\
\hline $\begin{array}{l}\text { Myrsine coriacea (Sw.) R.Br. ex Roem. \& } \\
\text { Schult. }\end{array}$ & 2 & & & 9046 \\
\hline \multicolumn{5}{|l|}{ Rosaceae } \\
\hline Prunus myrtifolia (L.) Urb. & 32 & 3 & & 9050 \\
\hline \multicolumn{5}{|l|}{ Salicaceae } \\
\hline Azara uruguayensis (Speg.) Sleumer & 1 & 1 & & 9022 \\
\hline \multicolumn{5}{|l|}{ Solanaceae } \\
\hline Solanum cassioides L.B.Sm. \& Downs & 1 & & & 9053 \\
\hline Solanum compressum L.B.Sm. \& Downs & & & 1 & 9054 \\
\hline Solanum pabstii L.B.Sm. \& Downs & 5 & & & 9055 \\
\hline Solanum paranense Dusén & 1 & & & 9056 \\
\hline \multicolumn{5}{|l|}{ Winteraceae } \\
\hline Drimys angustifolia Miers & 99 & 115 & 27 & 9032 \\
\hline Total & 375 & 322 & 198 & \\
\hline
\end{tabular}

${ }^{\bar{N} \text { Número de registro no LUSC - Herbário da Universidade do Estado de Santa Catarina }}$ 
A redução da riqueza e a substituição florística ao longo de gradiente altitudinal, representada pelo endemismo, é um padrão que ocorre em escala global (RAHBEK, 2005) e é o resultado de filtros ambientais que são impostos por fatores já destacados em outros estudos, como a baixa temperatura (OLIVEIRA FILHO; FONTES, 2000), os solos de baixa fertilidade (SCHEER; CURCIO; RODERJAN, 2011; MARCON et al., 2014) e a presença constante de neblina (HIGUCHI et al., 2013). Além disso, infere-se que, quando as espécies apresentam um nicho ecológico de baixa amplitude, os ambientes altomontanos podem ser exclusivos para as mesmas. Este aspecto cria uma condição de isolamento reprodutivo das espécies situadas nas áreas de elevada altitude, análoga a ilhas oceânicas que, em longo prazo, favorece o processo de especiação, o que pode explicar o endemismo observado nestas áreas (FALKENBERG, 2003). Assim, florestas altomontanas se tornam particularmente sensíveis em um cenário de mudanças climáticas, uma que vez que, com o aumento da temperatura global, ambientes com temperaturas reduzidas podem se tornar limitados, ocasionando a não disponibilidade de nichos para as espécies adaptadas a eles.

As curvas de acumulação de espécies (Figura 2) demonstraram que as amostragens foram suficientes para caracterizar as composições florísticas das áreas analisadas, uma vez que, segundo Kersten e Galvão (2011), com o aumento de $10 \%$ na área amostral, considera-se como suficiente um aumento de até 5\% em número de espécies. Na área "Estágio avançado 1" (Figura 2a) observa-se que, com o acréscimo das duas últimas parcelas $(10 \%$ da área amostrada), houve um incremento de apenas uma espécie $(3,0 \%$ da riqueza da área). Resultados semelhantes foram encontrados para as áreas "Estágio avançado 2" (Figura 2b) e "Estágio Inicial" (Figura 2c), que apresentaram, respectivamente, o incremento de 0,4 espécies (2,4\% da riqueza área) e 0,7 espécies $(5,0 \%$ da riqueza da área).
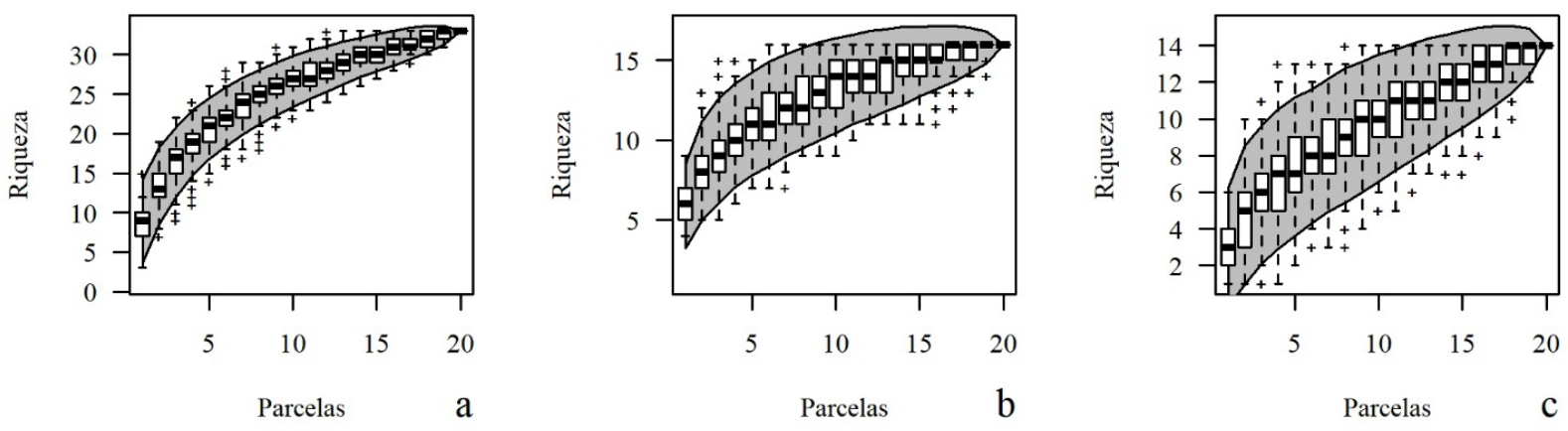

FIGURA 2: Curvas de acumulação de espécies para três fragmentos florestais em diferentes estágios sucessionais (a $=$ Estágio Avançado 1; $\mathrm{b}=$ Estágio Avançado 2; $\mathrm{c}=$ Estágio Inicial) em Urubici-SC.

FIGURE 2: Species accumulation curve for three forest fragments, in different successional stages $(\mathrm{a}=$ Advanced Stage 1; b = Advanced Stage 2; $c=$ Initial Stage), in the municipality of Urubici, SC state.

$\mathrm{Na}$ área "Estágio Avançado 1" foram amostrados 375 indivíduos distribuídos em 33 espécies; na área "Estágio Avançado 2", 322 indivíduos distribuídos em 16 espécies e na área "Estágio Inicial", 198 indivíduos e 14 espécies. Considerando a padronização pela rarefação (representada pela linha vertical no local de intersecção das curvas), não houve diferenças significativas entre as áreas "Estágio Inicial" e "Estágio Avançado 2", sendo a área "Estágio Avançado 1" distinta das demais (Figura 3). Ao contrário do observado em outros estudos (LETCHER; CHAZDON, 2009; ZANINI et al., 2014), não ficou evidenciada uma relação direta entre a riqueza de espécies e o estágio sucessional, já que a área em estágio inicial e uma das áreas em estágio tardio (Estágio Avançado 2) não diferiram quanto à riqueza, sendo somente na área "Estágio Avançado 1" observado maior número espécies. Este resultado sugere a importância de diferenças ambientais locais na determinação do número de espécies em cronossequências florestais, reforçando a ideia da complexidade do processo de sucessão, de 
forma que a trajetória de desenvolvimento da vegetação pode apresentar variações em escala local (NORDEN et al., 2015; ARROYO-RODRÍGUEZ et al., 2017). Neste sentido, infere-se que as diferenças na riqueza entre as áreas em avançado estágio sucessional estejam relacionadas às diferenças locais entre elas. Como indicado na descrição das áreas na metodologia, a partir de observações de campo, foi constatada a maior presença de clareiras na área "Estágio Avançado 1", o que pode resultar em uma maior heterogeneidade do mosaico silvigenético, e a presença de um pequeno curso d'água na área "Estágio Avançado 2", que pode resultar em um ambiente com condições ambientais mais restritivas nesta área.

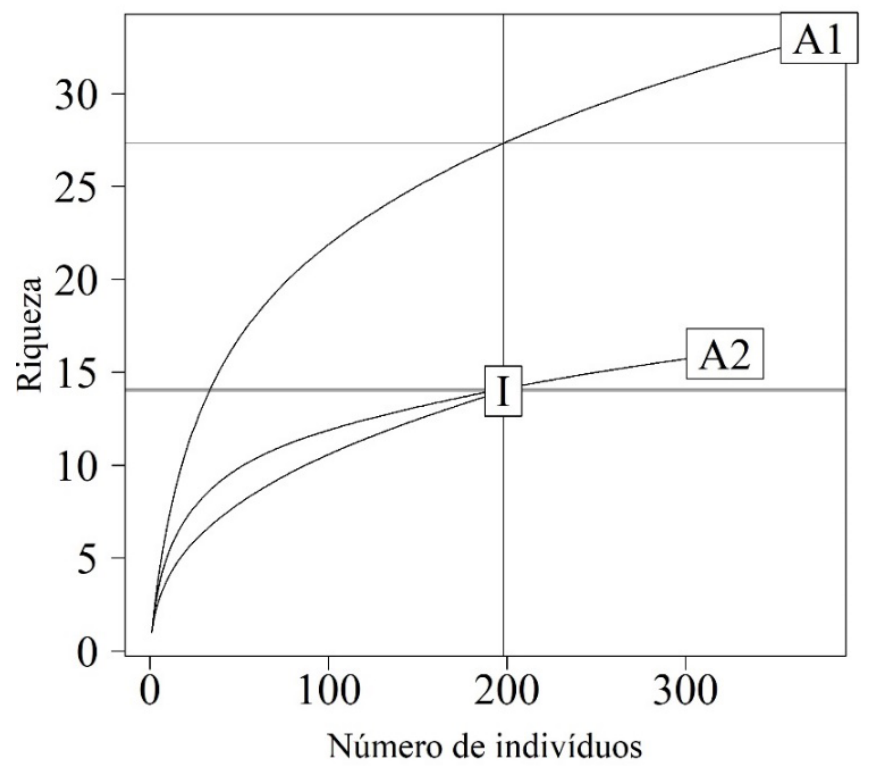

FIGURA 3: Curvas de rarefação para as riquezas amostradas em três fragmentos florestais, em diferentes estágios de sucessão (A1= Estágio Avançado 1; A2= Estágio Avançado 2; I= Estágio Inicial) em Urubici-SC. A linha vertical no local da interseç̧ão das curvas indica a padronização da riqueza pelo número de indivíduos.

FIGURE 3: Richness rarefaction curves for three forest fragments, in different successional stages (A1= Advanced Stage 1; A2= Advanced Stage 2; I= Initial Stage), in the municipality of Urubici, SC. The intersection vertical line indicates the number of individuals' standardized richness.

Apesar de a riqueza de espécies não possuir relação direta com os estágios sucessionais, considerando que, como discutido anteriormente, outros aspectos ambientais podem ter sido determinantes, a densidade de indivíduos refletiu a distinção entre os estágios. Assim, na área mais inicial foram amostrados menos indivíduos arbóreos (198) que as demais áreas (mais do que 300 indivíduos). No entanto, este padrão difere do que Norden et al. (2015), avaliando a sucessão por meio de parcelas permanentes em florestas neotropicais, concluíram. Estes autores observaram que fatores ambientais específicos são, também, mais relevantes que o tempo pós-distúrbio para as mudanças estruturais. Considerando estes resultados antagônicos, entre o que foi observado neste estudo para florestas subtropicais de altitude e o que é relatado na literatura para florestas tropicais, infere-se que a trajetória sucessional pode apresentar, também, variações em função do tipo de fitofisionomia, clima e domínio fitogeográfico.

Entre todas as áreas, houve baixo compartilhamento florístico (duas espécies), com maior compartilhamento de espécies entre as áreas "Estágio Avançado 1" e "Estágio Avançado 2" (14 espécies) (Figura 4). O resultado da análise multivariada de NMDS para ordenação das áreas (stress $=0,13$ ) confirmou esse padrão, com a formação de grupos florístico-estruturais distintos em cada área (Figura 5). $\mathrm{O}$ eixo 1 da NMDS separou os estágios sucessionais, com o lado esquerdo do gráfico contendo as parcelas das 
áreas em estágios avançados, representadas, principalmente, por espécies mais tardias, como as da família Myrtaceae, além de Dicksonia sellowiana e Ocotea porosa, e com o lado direito contendo a área em estágio inicial, com representação de pioneiras como Baccharis uncinella e Mimosa scabrella. Já o eixo 2 separou as parcelas das duas áreas em estágio avançado (Estágio Avançado 2 acima do gráfico e Estágio Avançado 1 abaixo).

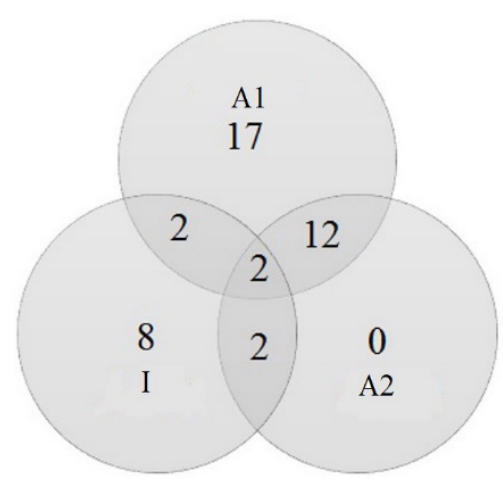

FIGURA 4: Diagrama de Venn das espécies amostradas em três fragmentos florestais, em diferentes estágios sucessionais (A1 = Estágio Avançado 1; A2 = Estágio Avançado 2; I = Estágio Inicial), em Urubici-SC.

FIGURE 4: Venn diagram of sampling species in three forest fragments, in different successional stages $(\mathrm{A} 1=$ Advanced Stage 1; A2 = Advanced Stage 2; I = Initial Stage), in the municipality of Urubici, SC state.

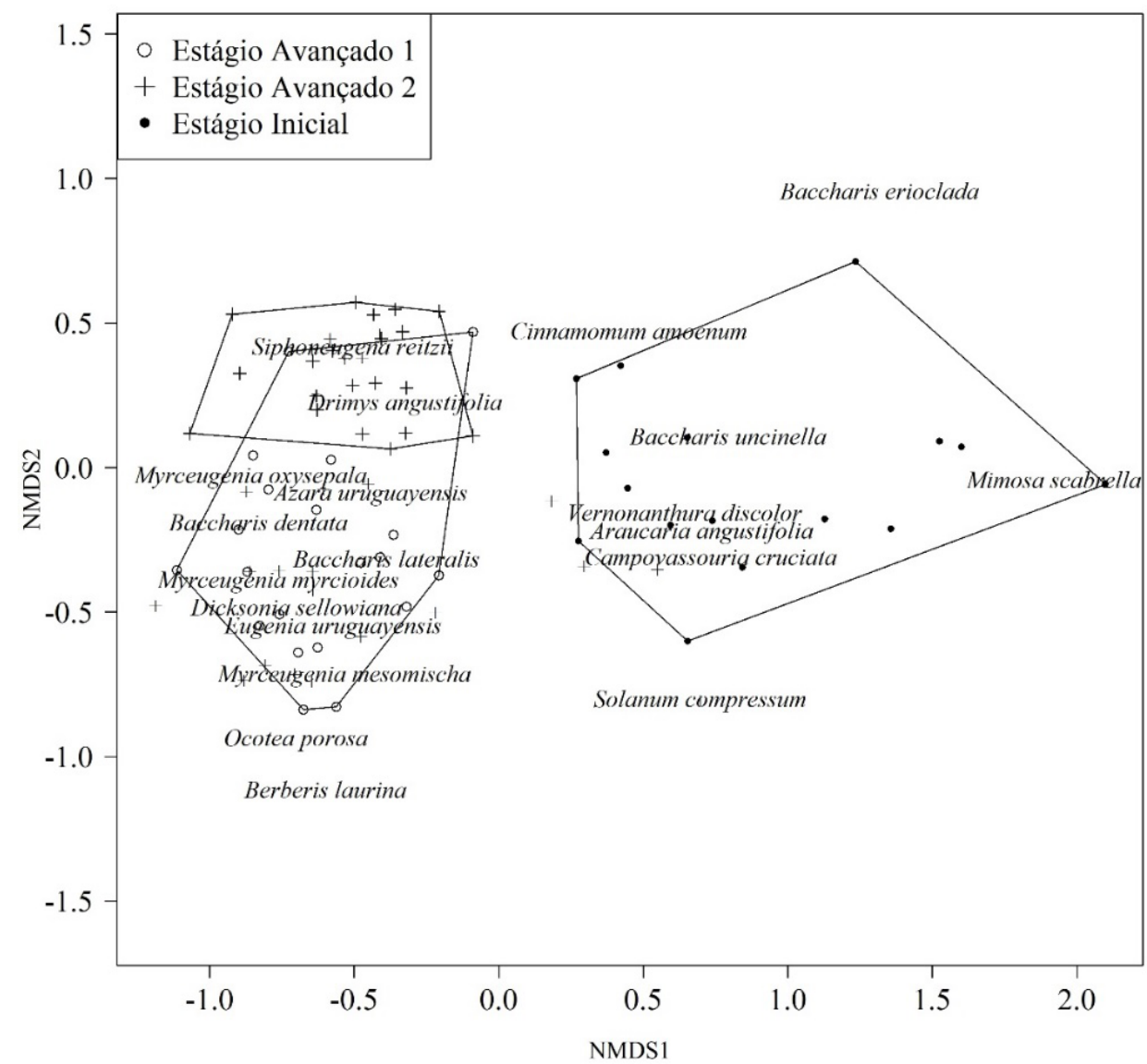

FIGURA 5: Ordenação produzida por Escalonamento Multidimensional Não Métrico (NMDS) para três fragmentos florestais, em diferentes estágios sucessionais, em Urubici-SC.

FIGURE 5: Ordination produced by Non Metric Dimensional Scaling (NMDS) for three forest fragments, in different successional stages, in the municipality of Urubici, SC state. 
Assim, infere-se que a composição florístico-estrutural foi influenciada pela interação entre o estágio sucessional e as condições locais das áreas, reforçando a ideia, como observado por Norden et al. (2015), de que a trajetória sucessional é influenciada pela complexa interação entre vários fatores (e.g. condições ambientais, paisagem do entorno, histórico de perturbação), além do tempo pós-distúrbio. Enquanto o eixo 1 da NMDS evidenciou a importância do estágio sucessional, indicando maior similaridade das áreas "Estágio Avançado 1" e "Estágio Avançado 2" e maior distinção da área "Estágio Inicial", o eixo 2 evidenciou as diferenças locais entre as áreas, distinguindo as áreas em avançado estágio sucessional, apesar da elevada sobreposição de espécies observadas entre elas, indicando maiores distinções estruturais.

Nos setores em avançado estágio de sucessão, as espécies que se destacaram como indicadoras foram Myrceugenia myrcioides (valor indicador de 0,8500 na área "Estágio Avançado 1") e Crinodendron brasiliense (valor indicador de 0,8323 na área "Estágio Avançado 2") (Tabela 2), além de um conjunto expressivo de espécies de Myrtaceae e outras típicas de florestas nebulares. Já no setor inicial ("Estágio Inicial"), as espécies que se destacaram como indicadoras foram, principalmente, pioneiras, como Baccharis uncinella $(0,6500)$ e Mimosa scabrella $(0,6500)$. Destaca-se que tanto as espécies indicadoras da família Myrtaceae, como a maioria das espécies típicas nebulares, são zoocóricas, reforçando a importância das interações com os animais na dispersão de propágulo das florestas mais tardias nebulares, enquanto na área inicial predominou espécies anemocóricas, como as da família Asteraceae. Ressalta-se que a presença de Araucaria angustifolia como indicadora da área em estágio inicial reflete a interação entre o aspecto sucessional e ambiental (menor altitude e ausência de condição nebular), visto que esta é uma espécie colonizadora de áreas abertas (DUARTE et al., 2006; RIBEIRO et al., 2013) e sua abundância não é elevada em florestas nebulares (MARCON et al., 2014), como é o caso das áreas "Estágio Avançado 1" e "Estágio Avançado 2".

TABELA 2: Espécies arbóreas indicadoras para três fragmentos florestais, em diferentes estágios sucessionais $(\mathrm{A} 1=$ Estágio Avançado 1; A2 = Estágio Avançado 2; I = Estágio Inicial), em Urubici-SC.

TABLE 2: Indicator tree species for three forest fragments in, in different successional stages $(A 1=$ Advanced Stage 1; A2 = Advanced Stage 2; I = Initial Stage), in the municipality of Urubici, SC state.

\begin{tabular}{cccc}
\hline Espécies & Áreas & $\begin{array}{c}\text { Valor } \\
\text { indicador }\end{array}$ & $P$ \\
\hline Myrceugenia myrcioides & A1 & 0,85 & 0,001 \\
Prunus myrtifolia & A1 & 0,594 & 0,001 \\
Eugenia uruguayensis & A1 & 0,55 & 0,001 \\
Myrceugenia oxysepala & A1 & 0,539 & 0,002 \\
Myrceugenia mesomischa & A1 & 0,5 & 0,001 \\
Weinmannia paulliniifolia & A1 & 0,409 & 0,004 \\
Dicksonia sellowiana & A1 & 0,4 & 0,001 \\
Myrceugenia miersiana & A1 & 0,35 & 0,001 \\
Citronella paniculata & A1 & 0,25 & 0,002 \\
Crinodendron brasiliense & A2 & 0,832 & 0,001 \\
Siphoneugena reitzii & A2 & 0,748 & 0,001 \\
Myrceugenia regnelliana & A2 & 0,572 & 0,001 \\
Drimys angustifolia & A2 & 0,453 & 0,002 \\
Myrceugenia glaucescens & A2 & 0,246 & 0,001 \\
Baccharis uncinella & I & 0,65 & 0,001 \\
Mimosa scabrella & I & 0,65 & 0,001 \\
Araucaria angustifolia & I & 0,405 & 0,002 \\
Campovassouria cruciata & I & 0,2 & 0,027 \\
\hline
\end{tabular}


A resolução da árvore filogenética não teve influência significativa sobre as métricas calculadas, indicando a adequabilidade das análises para interpretação. Para a área "Estágio Avançado 1" (Figura 6a), a espécie mais basal de angiosperma foi Drimys angustifolia, seguida por Persea willdenovii, Ocotea pulchella e Ocotea porosa. O clado de maior riqueza foi Malvids, com 11 espécies, sendo todas pertencentes à família Myrtaceae. O gênero mais representativo foi Myrceugenia, com sete espécies. O valor de MPD (distância filogenética média em milhões de anos) foi de 206,80 milhões de anos e NRI de 0,57, com as espécies distribuídas de forma aleatória na árvore filogenética $(p>0,05)$. Na área "Estágio Avançado 2" (Figura 6b), a espécie mais basal de angiosperma também foi Drimys angustifolia, seguida por Ocotea pulchella. Os clados de maior riqueza foram Fabids e Malvids, com cinco espécies cada, sendo as famílias Celastraceae, Cunoniaceae, Elaeocarpaceae, Rosaceae e Salicaceae pertencentes à Fabids, e quatro espécies de Myrtaceae e uma de Anacardiaceae, representando Malvids. O gênero de maior riqueza foi Myrceugenia, com três espécies. O valor de MPD foi de 199,26 milhões de anos e o NRI de 0,80, com um padrão aleatório das espécies na árvore filogenética $(p>0,05)$. Na área "Estágio Inicial" (Figura 6c), a espécie mais basal de angiosperma foi Drimys angustifolia, seguida por Cinnamomum amoenum. O clado de maior riqueza foi Campanulids, com sete espécies, sendo seis pertencentes à família Asteraceae e uma à Aquifoliaceae. $\mathrm{O}$ gênero mais expressivo foi Baccharis, com três espécies. O valor de MPD foi de 149,80 milhões de anos e o NRI de 2,14, o que caracteriza um agrupamento filogenético de espécies $(p<0,05)$.
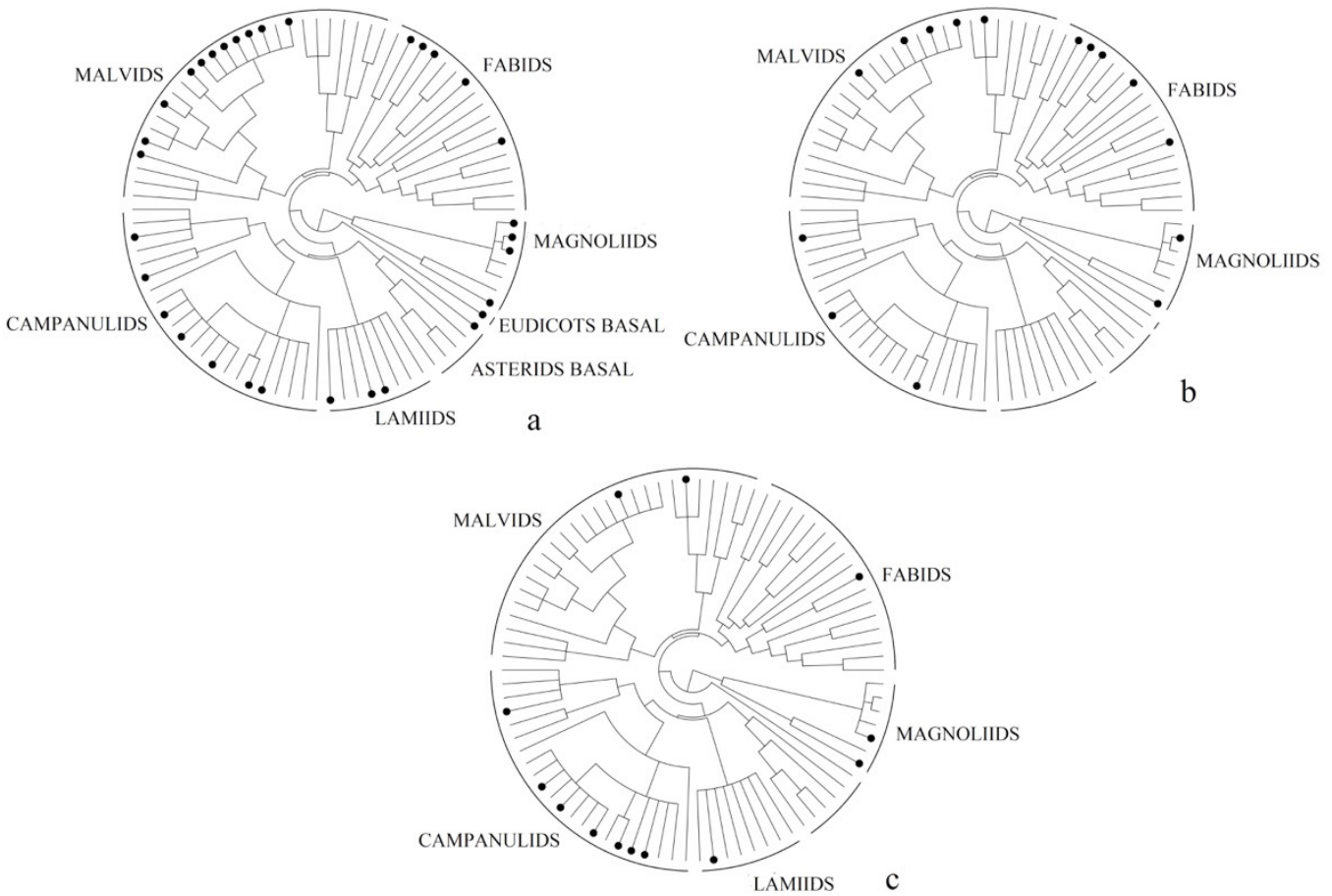

FIGURA 6: Árvores filogenéticas construídas a partir das espécies amostradas em três fragmentos florestais, em diferentes estágios sucessionais ( $\mathrm{a}=$ Estágio Avançado $1 ; \mathrm{b}=$ Estágio Avançado 2; $\mathrm{c}=$ Estágio Inicial) em Urubici-SC.

FIGURE 6: Phylogenetic trees constructed from sampled tree species in three forest fragments, in different successional stages $(\mathrm{a}=$ Advanced Stage $1 ; \mathrm{b}=$ Advanced Stage 2; $\mathrm{c}=$ Initial Stage $)$ in the municipality of Urubici, SC state.

Com esses resultados, infere-se que a importância do estágio sucessional também ficou evidenciado na organização da estrutura filogenética das comunidades, com a área em início de sucessão apresentando um padrão de agrupamento filogenético e as áreas tardias, com padrões aleatórios. O agrupamento filogenético na área em estágio inicial pode ser explicado pela elevada abundância e riqueza de espécies pertencentes à Asteraceae. Conforme destacado por Prinzing et al. (2017), é comum que espécies de alguns 
clados específicos ocorram preferencialmente em um determinado tipo de habitat. De fato, Asteraceae, assim como também observado por Duarte (2011) em pequenos capões florestais em meio a uma matriz campestre no Rio Grande do Sul, se destaca por apresentar espécies exigentes em elevada incidência lumínica e com dispersão anemocórica, o que confere uma grande capacidade de colonização em áreas abertas. Assim, os resultados sugerem que as características ecológicas que conferem o caráter pioneiro das espécies presentes na área em estágio inicial de sucessão sejam filogeneticamente conservadas, ou seja, herdadas de um antepassado em comum. Por outro lado, o padrão aleatório da estruturação filogenética nas áreas "Estágio Avançado 1" e "Estágio Avançado 2" demonstra a importância da função das espécies pioneiras como facilitadoras para a colonização de espécies pertencentes a outros clados filogenéticos. Apesar de este mesmo tipo de resultado também ter sido observado em outros estudos (LETCHER et al., 2012; MARCILIO-SILVA; PILLAR; MARQUES, 2016), ressalta-se que a literatura não indica que este padrão possa ser generalizado (DUARTE, 2011; CHANG; ZHOU; PENG, 2015; SCHWEIZER et al., 2015), demonstrando que as forças ecológicas condicionadoras de cada fase do processo de sucessão vegetacional podem variar em função do tipo de vegetação, histórico de uso do solo, estrutura da paisagem e condições ambientais locais.

\section{CONCLUSÕES}

O presente estudo enfatizou o caráter único da vegetação das áreas de elevada altitude da região Sul (baixa riqueza e endemismo) e ilustrou a complexidade do processo de sucessão. Por meio da existência de espécies pertencentes a guildas de regeneração distintas, observaram-se diferenças florístico-estruturais e de estrutura filogenética entre as áreas em estágio sucessional inicial e avançado. Na fase inicial, no qual existiu o agrupamento filogenético das espécies, ficou evidenciada a importância do grupo das pioneiras, com destaque para aquelas pertencentes à família Asteraceae, que desempenham papel-chave de facilitação para a chegada de espécies tardias. Nas fases tardias, em que o padrão filogenético foi aleatório, Mytaceae foi a família de maior representatividade de espécies, e o fato das mesmas apresentarem dispersão zoocórica reforça a importância das relações biológicas ao longo do tempo.

\section{AGRADECIMENTOS}

Ao Dr. Marcos Sobral, pelo auxílio na identificação das espécies de Myrtaceae. À FAPESC (Fundação de Amparo à Pesquisa e Inovação do Estado de Santa Catarina), pela concessão de bolsa à primeira autora do trabalho e pelo apoio financeiro por meio do Edital PAP/UDESC. Ao CNPq, pelo financiamento do projeto e pela concessão de bolsa de produtividade para o segundo e o terceiro autores. Ao Instituto Chico Mendes de Conservação da Biodiversidade, pela permissão para execução do trabalho no Parque Nacional de São Joaquim. À equipe do PARNA São Joaquim, em especial ao MSc. Michel Omena, pelo apoio logístico na execução do trabalho.

\section{REFERÊNCIAS}

ANGIOSPERM PHYLOGENY GROUP. An update of the Angiosperm Phylogeny Group classification for the orders and families of flowering plants: APG III. Botanical Journal of the Linnean Society, Londres, v. 161, n. 2, p. 105-121, 2009.

ARROYO-RODRÍGUEZ, V. et al. Multiple successional pathways in human-modified tropical landscapes: new insights from forest succession, forest fragmentation and landscape ecology research. Biological Reviews, Hoboken, v. 92, n. 1, p. 326-340, 2017.

BEGON, M.; TOWNSEND, C. R.; HARPER, J. L. Ecology: from individuals to ecosystems. Oxford: Blackwell, 2006. 738 p.

BELL, C. D.; SOLTIS, D. E.; SOLTIS, P. S. The age and diversification of the angiosperms re-revisited. American Journal of Botany, St. Louis, v. 97, n. 8, p. 1296-1303, 2010.

BELLO, F. et al. Decoupling phylogenetic and functional diversity to reveal hidden signals in community assembly. Methods in Ecology and Evolution, Hoboken, v. 8, n.10, p. 1200-1211, out. 2017. 
CHANG, X.; ZHOU, T.; PENG, S. Change in community phylogenetic structure of tropical forest along the southern coast of China during restoration. Ecosphere, Hoboken, v. 6, n. 9, p. 1-13, 2015.

CHAZDON, R. L. Chance and determinism in tropical forest succession. In: CARSON, W.; SCHNITZER, S. A. (Ed.). Tropical forest community ecology. Oxford: Wiley-Blackwell, 2008. p. 384-408.

CIANCIARUSO, M. V.; SILVA, I. A.; BATALHA, M. A. Diversidades filogenética e funcional: novas abordagens para a Ecologia de comunidades. Biota Neotropica, Campinas, v. 9, n. 3, p. 93-103, 2009.

DEXTER, K. G. et al. Dispersal assembly of rain forest tree communities across the Amazon basin. Proceedings of the National Academy of Sciences, Washington, v. 114, n. 10, p. 2645-2650, 2017.

DUARTE, L. S. et al. Role of nurse plants in Araucaria Forest expansion over grassland in south Brazil. Austral Ecology, Hoboken, v. 31, n. 4, p. 520-528, 2006.

DUARTE, L. S. Phylogenetic habitat filtering influences forest nucleation in grasslands. Oikos, Hoboken, v. 120, n. 2, p. 208-215, 2011.

DUFRANE, M.; LEGENDRE, P. Species assemblages and indicator species: the need for a flexible asymmetrical approach. Ecological Monographs, Hoboken, v. 67, n. 3, p. 345-366, 1997.

FALKENBERG, D. B. Matinhas nebulares e vegetação rupícola dos Aparados da Serra Geral (SC/RS), sul do Brasil. 2003. 594 f. Tese (Doutorado em Biologia Vegetal) - Universidade Estadual de Campinas, Campinas, 2003.

GASTAUER, M.; MEIRA NETO, J. A. A. An enhanced calibration of a recently released megatree for the analysis of phylogenetic diversity. Brazilian Journal of Biology, São Carlos, v. 76, n. 3, p. 619-628, 2016. GERHOLD, P. et al. Phylogenetic patterns are not proxies of community assembly mechanisms (they are far better). Functional Ecology, Hoboken, v. 29, n. 5, p. 600-614, 2015.

GOTELLI, N. J. Null model analysis of species co-occurrence patterns. Ecology, Hoboken, v. 81, n. 9, p. 2606-2621, 2000.

GUARIGUATA, M. R.; OSTERTAG, R. Neotropical secondary forest succession: changes in structural and functional characteristics. Forest Ecology and Management, Amsterdam, v. 148, n. 1-3, p. 185-206, 2001. HEDGES, S. B.; KUMAR, S. The time tree of life. New York: Oxford University Press, 2009. 18 p.

HIGUCHI, P. et al. Florística e estrutura do componente arbóreo e análise ambiental de um fragmento de Floresta Ombrófila Mista Alto-Montana no município de Painel, SC. Ciência Florestal, Santa Maria, v. 23, n. 1, p. 153-164, 2013.

HIGUCHI, P. et al. Influência de variáveis ambientais sobre o padrão estrutural e florístico do componente arbóreo, em um fragmento de Floresta Ombrófila Mista Montana em Lages, SC. Ciência Florestal, Santa Maria, v. 22, n. 1, p. 79-90, 2012.

HIJMANS, R. J. et al. Very high resolution interpolated climate surfaces for global land areas. International Journal of Climatology, Hoboken, v. 25, n. 15, p. 1965-1978, 2005.

IBGE. Manual técnico da vegetação brasileira. Rio de Janeiro: Fundação Instituto Brasileiro de Geografia e Estatística, 2012.271 p.

KEMBEL, S. W. et al. Picante: R tools for integrating phylogenies and ecology. Bioinformatics, Oxford, v. 26, n. 11, p. 1463-1464, 2010.

KERSTEN, R. A.; GALVÃO, F. Suficiência amostral em inventários florísticos e fitossociológicos. In: FELFILI, J. M. et al. (Ed.). Fitossociologia no Brasil: métodos e estudos de casos. Viçosa, MG: Editora UFV, 2011. p. 153-176.

LETCHER, S. G.; CHAZDON, R. L. Rapid recovery of biomass, species richness, and species composition in a forest chronosequence in northeastern Costa Rica. Biotropica, Hobeken, v. 41, n. 5, p. 608-617, 2009. LETCHER S. G. et al. Phylogenetic community structure during succession: evidence from three Neotropical forest sites. Perspectives in Plant Ecology, Evolution and Systematics, Amsterdam v. 14, n. 2, p. 79-87, 2012.

MARCILIO-SILVA, V.; PILLAR, V. D.; MARQUES, M. C. M. Functional turnover and community assemblage during tropical forest succession. Community Ecology, Budapeste, v. 17, n. 1, p. 88-97, 2016. MARCON, A. K. et al. Variação florístico-estrutural em resposta à heterogeneidade ambiental em uma floresta nebular em Urubici, Planalto Catarinense. Scientia Forestalis, Piracicaba, v. 42, n. 103, p. 439-450, 2014.

NASCIMENTO, A. R. T.; LONGHI, S. J.; BRENA, A. D. Estrutura e padrões de distribuição espacial de 
espécies arbóreas em uma amostra de Floresta Ombrófila Mista em Nova Prata, RS. Ciência Florestal, Santa Maria, v. 11, n. 1, p. 105-119, 2001.

NORDEN, N. et al. Successional dynamics in Neotropical forests are as uncertain as they are predictable. PNAS, Washington, v. 112, n. 26, p. 8013-8018, 2015.

OKSANEN, J. et al. Vegan: community ecology package. [s. 1.]: R package version, 2009.

OLIVEIRA FILHO, A. T.; FONTES, M. A. L. Patterns of floristic differentiation among Atlantic forests in south-eastern Brazil, and the influence of climate. Biotropica, Hoboken, v. 32, n. 4b, p. 793-810, 2000. PRINZING, A. et al. Benefits from living together? Clades whose species use similar habitats may persist as a result of eco-evolutionary feedbacks. New Phytologist, Hoboken, v. 213, n. 1, p. 66-82, 2017.

R DEVELOPMENT CORE TEAM. R: a language and environment for statistical computing. [2016]. Disponível em: <http://www.R-project.org> Acesso em: 16 out. 2016.

RAHBEK, C. The role of spatial scale and the perception of largescale species-richness patterns. Ecology Letters, Hoboken, v. 8, n. 2, p. 224-239, 2005.

RIBEIRO, T. M. et al. Fitossociologia de uma floresta secundária com Araucaria angustifolia (Bertol.) O. Kuntze na Estação Ecológica de Bananal, Bananal-SP. Floresta e Ambiente, Seropédica, v. 20, n. 2, p. 159-172, 2013.

ROBERTS, D. W. Labdsv: ordination and multivariate analysis for ecology. [2016]. Disponível em: $<$ http://cran.r-project.org/package=labdsv $>$ Acesso em: 16 out. 2016.

SCHEER, M. B.; CURCIO, G. R.; RODERJAN, C. V. Funcionalidades ambientais de solos altomontanos na Serra da Igreja, Paraná. Revista Brasileira de Ciência do Solo, Viçosa, MG, v. 35, n. 4, p. 1113-1126, 2011.

SCHWEIZER, D. et al. Phylogenetic patterns of Atlantic forest restoration communities are mainly driven by stochastic, dispersal related factors. Forest Ecology and Management, Amsterdam, v. 354, n. 15, p. 300-308, 2015.

SWENSON, N. G. Phylogenetic resolution and quantifying the phylogenetic diversity and dispersion of communities. PloS one, São Francisco, v. 4, n. 2, p. e4390, 2009.

VARJABEDIAN, R. Lei da Mata Atlântica: retrocesso ambiental. Estudos Avançados, São Paulo, v. 24, n. 68, p. $147-160,2010$.

WEBB, C. O. Exploring the phylogenetic structure of ecological communities: an example for rain forest trees. The American Naturalist, Chicago, v. 156, n. 2, p. 145-155, 2000.

WEBB, C. O.; ACKERLY, D. D.; KEMBEL, S. W. Phylocom: software for the analysis of phylogenetic community structure and trait evolution. Bioinformatics, Oxford, v. 24, n. 18, p. 2098-2100, 2008.

WEBB, C. O.; DONOGHUE, M. J. Phylomatic: tree assembly for applied phylogenetics. Molecular Ecology Resources, Hoboken, v. 5, n. 1, p. 181-183, 2004.

WEBB, C. O. et al. Phylogenies and community ecology. Annual Review of Ecology and Systematics, Palo Alto, v. 33, p. 475-505, 2002.

WIRTH, C. et al. Old-growth forest definitions: a pragmatic view. In: WIRTH, C.; GLEIXNER, G.; HEIMANN, M. (Ed.). Old-growth forests: function, fateand value. New York: Springer, 2009. p. 11-33.

ZANINI, K. J. et al. Atlantic rain forest recovery: successional drivers of floristic and structural patterns of secondary forest in Southern Brazil. Journal of Vegetation Science, Hoboken, v. 25, n. 4, p. 1056-1068, 2014. 